\title{
Sphingosine-1-phosphate induces VEGF-C expression through a MMP-2/FGF-1/FGFR-1-dependent path- way in endothelial cells in vitro
}

\author{
Chi-hao CHANG ${ }^{1, \#}$, Yuan-li HUANG ${ }^{2, \#, ~ M i n g-k w a n g ~ S H Y U ~}{ }^{3, \#}$, Shee-uan CHEN ${ }^{3}$, Chih-hsin LIN', Tsai-kai JU ${ }^{4,5}$, JenHer LU ${ }^{6, *}$, \\ Hsinyu LEE ${ }^{1,7,8,9,10, *}$

\begin{abstract}
${ }^{1}$ Institute of Zoology, National Taiwan University, Taipei, Taiwan, China; ${ }^{2}$ Department of Biotechnology, Asia University, Taichung, Taiwan, China; ${ }^{3}$ Department of Obstetrics and Gynecology, National Taiwan University Hospital, Taipei, Taiwan, China; ${ }^{4}$ Instrumentation Center, National Taiwan University, Taipei, Taiwan, China; ${ }^{5}$ Technology Commons, College of Life Science, National Taiwan University, Taipei, Taiwan, China; ${ }^{6}$ Department of Pediatrics and Pediatric Cardiology, Veterans General Hospital-Taipei, National Yang Ming University, Taipei, Taiwan, China; ${ }^{7}$ Department of Life Science, National Taiwan University, Taipei, Taiwan, China; ${ }^{8}$ Center for Biotechnology, National Taiwan University, Taipei, Taiwan, China; ${ }^{9}$ Angiogenesis Research Center, National Taiwan University, Taipei, Taiwan, China;

${ }^{10}$ Research Center for Developmental Biology and Regenerative Medicine, National Taiwan University, Taipei, Taiwan, China
\end{abstract}

Aim: To investigate whether sphingosine-1-phosphate (S1P), a potent angiogenic factor, induced vascular endothelial growth factor-C (VEGF-C) expression in endothelial cells in vitro and to examine its underlying mechanisms.

Methods: Human umbilical vein endothelial cells (HUVECs) were examined. VEGF-C mRNA expression in the cells was assessed using real-time PCR. VEGF-C protein and FGFR-1 phosphorylation in the cells were measured with ELISA. RNA interference was used to downregulate the expression of matrix metalloproteinase-2 (MMP-2), fibroblast growth factor-1 (FGF-1) and FGF receptor-1 (FGFR-1). Results: Incubation of HUVECs with $\operatorname{S1P}(1,5$, and $10 \mu \mathrm{mol} / \mathrm{L})$ significantly increased VEGF-C expression. The effect was blocked by pretreatment with the MMP inhibitor GM6001 or the FGFR inhibitor SU5402, but not the EGFR inhibitor AG1478. The effect was also blocked in HUVECs that were transfected with FGFR-1 or MMP-2 siRNA. Furthermore, incubation of HUVECs with S1P (5 $\mu$ mol/L) significantly increased FGFR-1 phosphorylation, which was blocked by GM6001. Moreover, knockdown of FGF-1, not FGF-2, in HUVECs with siRNAs, blocked S1P-induced VEGF-C expression.

Conclusion: S1P induces VEGF-C expression through a MMP-2/ FGF-1/FGFR-1-dependent pathway in HUVECs.

Keywords: sphingosine-1-phosphate; VEGF-C; matrix metalloproteinase-2; fibroblast growth factor-1; fibroblast growth factor receptor-1; GM6001; SU5402; transactivation; human umbilical vein endothelial cells; RNA interference

Acta Pharmacologica Sinica (2013) 34: 360-366; doi: 10.1038/aps.2012.186; published online 4 Feb 2013

\section{Introduction}

Sphingosine-1-phosphate (S1P) is a low molecular weight lysophospholipid (LPL) composed of a sphingoid backbone and a phosphate group. It is a normal constituent of human plasma and serum, and its respective levels in plasma and serum are approximately 190 and $480 \mathrm{pmol} / \mathrm{mL}^{[1]}$. S1P acts as an extracellular regulator by binding to 5 different G-protein-

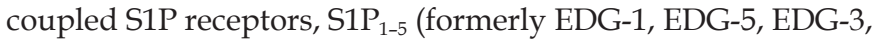
EDG-6, and EDG-8), to regulate multiple biological processes,

\footnotetext{
\#These authors contributed equally to this work.

* To whom correspondence should be addressed.

E-mail hsinyu@ntu.edu.tw (Hsinyu LEE); jenherlu@gmail.com (JenHer LU)

Received 2012-07-30 Accepted 2012-12-12
}

including cell proliferation, migration, apoptosis, calcium homeostasis, angiogenesis, and vascular maturation ${ }^{[2]}$.

The progression of angiogenesis is regulated by various growth factors, such as vascular endothelial growth factors (VEGFs), fibroblast growth factors (FGFs), platelet-derived growth factors (PDGFs), angiopoietins, hepatocyte growth factor (HGF), and insulin-like growth factors (IGFs) ${ }^{[3]}$. Among these stimulators, VEGF-C has been characterized as an angiogenic and lymphangiogenic growth factor ${ }^{[4]}$, and has been shown to regulate these processes through binding to VEGF receptors (VEGFR) 2 and 3. S1P is known to be a potent angiogenic factor that regulates endothelial cell proliferation, migration, and differentiation. S1P has been shown to induce human umbilical vein endothelial cell (HUVEC) tube forma- 
tion both in vitro and in vivo ${ }^{[5]}$. Furthermore, neutralization of extracellular S1P significantly inhibits angiogenesis, tumor growth, and metastasis ${ }^{[6]}$, suggesting that S1P is an important regulator of angiogenesis. However, the role of S1P in VEGF-C expression in endothelial cells has not been investigated.

In the present study, we demonstrated that S1P enhances VEGF-C mRNA and protein expression in HUVECs and these enhancement effects were mediated through matrix metalloproteinase-2 (MMP-2), FGF-1, and FGFR-1.

\section{Materials and methods Reagents}

S1P, fatty acid-free bovine serum albumin (BSA), collagenase I, and gelatin were purchased from Sigma-Aldrich (St Louis, MO, USA). Medium 199 and fetal bovine serum (FBS) were purchased from Hyclone (Logan, UT, USA), and endothelial growth medium (EGM) was purchased from Cell Application (San Diego, CA, USA). Trypsin-EDTA was purchased from Gibco BRL (Grand Island, NY, USA). Penicillin and streptomycin were purchased from Invitrogen (Carlsbad, CA, USA). AG1478 and GM6001 were purchased from Calbiochem (La Jolla, CA, USA). Human recombinant FGF-2 was obtained from R\&D Systems (Minneapolis, MN, USA). FGFR-1 (L-003131-00-0005) and FGF-2 (L-006695-00-0005) small interfering (si)RNAs were obtained from Thermo Scientific Dharmacon (Lafayette, CO, USA). MMP-2 (sc-29398) and control siRNAs (sc-37007) were purchased from Santa Cruz Biotechnology (Santa Cruz, CA, USA). The HUVEC electroporation kit (VPB-1002) was purchased from Amaxa (Gaithersbug, MD, USA). The sequence of FGF-1 short-hairpin (sh)RNA (TRCN0000222593) for targeting the endogenous gene was 5'-CCTGATAACAAGCAAGGATAT-3', and it was obtained from the National RNAi Core Facility Platform of Academia Sinica (Taipei, Taiwan, China).

\section{Cell culture}

HUVECs were isolated from fresh umbilical cords using $0.1 \%$ collagenase I (Sigma) in cord buffer (136.9 mmol/L NaCl, 4 $\mathrm{mmol} / \mathrm{L} \mathrm{KCl}, 10 \mathrm{mmol} / \mathrm{L}$ HEPES, and $11.1 \mathrm{mmol} / \mathrm{L}$ glucose at $\mathrm{pH} 7.65$ ) in a $37^{\circ} \mathrm{C}$ incubator for $20 \mathrm{~min}$. After elution, HUVECs were collected by centrifugation and cultured on $1 \%$ gelatin-coated 10-cm plates containing M199 supplemented with 20\% (v/v) FBS, 20\% (v/v) EGM, $100 \mathrm{U} / \mathrm{mL}$ penicillin, and $100 \mathrm{mg} / \mathrm{mL}$ streptomycin. Cells underwent one passage weekly and were subcultured after trypsinization. Cells from passages 2-4 were used in these experiments.

\section{Determination of FGFR-1 phosphorylation using enzyme-linked immunosorbent assay (ELISA)}

Levels of phosphorylated FGFR-1 were measured using the phospho-FGFR-1 kit (R\&D Systems) following the manufacturer's instructions. Briefly, serum-starved HUVECs were pretreated with GM6001, followed by S1P $(1 \mu \mathrm{mol} / \mathrm{L})$ or FGF-2 (20 ng/mL) treatment for $1 \mathrm{~min}$. Cells were lysed in lysis buffer containing $1 \mathrm{mmol} / \mathrm{L}$ activated sodium orthova- nadate, $10 \mu \mathrm{g} / \mathrm{mL}$ aprotinin, and $10 \mu \mathrm{g} / \mathrm{mL}$ leupeptin. Cell lysates were centrifuged at $2000 \times g$ for $5 \mathrm{~min}$, and the supernatants were transferred into clean Eppendorf tubes. Before use, sample protein concentrations were quantified using the Bradford assay. The capture antibody specific for phosphorylated FGFR-1 was pre-coated on the ELISA plates and phosphoFGFR-1 levels were detected by the addition of a detection antibody. The optical density of each well was immediately determined using a microplate reader set to a wavelength of $450 \mathrm{~nm}$.

Determination of VEGF-C protein expression using enzyme-linked immunosorbent assay (ELISA)

HUVECs were treated with S1P for $8 \mathrm{~h}$. The conditioned medium was collected and VEGF-C levels were measured using a human VEGF-C ELISA kit (R\&D Systems) following the manufacturer's instructions. In brief, the capture antibody specific for VEGF-C was pre-coated on the ELISA plates and VEGF-C levels were detected by the addition of a detection antibody. The optical density of each well was immediately determined using a microplate reader set to a wavelength of $450 \mathrm{~nm}$.

\section{siRNA and shRNA electroporation}

HUVECs were transfected with siRNA using a nucleofector apparatus and HUVEC transfection kit (Amaxa Biosystems, Cologne, Germany). Electroporation procedures were followed by an optimization protocol for HUVECs, and the V-01 program of the nucleofector apparatus was used for transfection. HUVECs were trypsinized and collected by centrifugation. Cells $\left(5 \times 10^{5}\right)$ were resuspended in $100 \mu \mathrm{L}$ of supplemented HUVEC Nucleofector ${ }^{\mathrm{TM}}$ solution (Amaxa Biosystems) and electroporated with 30 pmol siRNA or shRNA. Transfected cells were then seeded in 6-well plates, and used after overnight incubation.

\section{RNA isolation and reverse transcription (RT)}

Total RNA was extracted from HUVECs using the TRIzol reagent (Invitrogen, Carlsbad, CA, USA). Complementary DNA (cDNA) was synthesized with $1 \mu \mathrm{g}$ total RNA using the Toyobo RT-polymerase chain reaction (PCR) kit (Toyobo, Osaka, Japan).

\section{Real-time PCR}

Real-time PCR was performed using the iCycler iQ real-time detection system (Bio-rad, Hercules, CA, USA) with SYBRGreen I (Thermo, Rockford, IL, USA) as the fluorescent dye, which enabled real-time detection of PCR products according to the manufacturer's protocol. Gene-specific primers were used, and the specificity was confirmed by meltingcurve detection following real-time PCR. Cycling conditions were $95^{\circ} \mathrm{C}$ for $3 \mathrm{~min}$, followed by 40 cycles of $95^{\circ} \mathrm{C}$ for $30 \mathrm{~s}, 60^{\circ} \mathrm{C}$ for $30 \mathrm{~s}$, and $72^{\circ} \mathrm{C}$ for $30 \mathrm{~s}$. For quantification, the target gene was normalized to the GAPDH internal standard gene. Primers for real-time PCR were as follows: human GAPDH (5'-AAGGTGAAGGTCGGAGTC-3' and 
5'-TGTAGTTGAGGTCAATGAAAGG-3'), VEGF-C (5'-CACACTTCCTGCCGATGC-3' and 5'-GTTCGCTGCCTGACACTG3'), MMP-2 (5'-TTGGTGGGAACTCAGAAGG-3' and 5'-CACTTGCGGTCATCATCG-3'), FGFR-1 (5'-AGATCCGGTCAAATAATGCC-3' and 5'-CCTGGTGACAGAGGACAATG-3'), FGFR-2 (5'-AACGGGAAGGAGTTTAAGCA-3' and 5'-CTTGTCAGATGGGACCACAC-3'), FGFR-3 (5'-AGGCCATCGGCATTGACA-3' and 5'-GCATCGTCTTTCAGCATCTTCAC-3'), FGFR-4 (5'-GTGTGTACACCCGGTCAAAC-3' and 5'-GCGTCCACCACATTGACTAC-3'), FGF-1 (5'-GATGGCACAGTGGATGGGAC-3' and 5'-AAGCCCGTCGGTGTCCATGG-3'), and FGF-2 (5'-GCTCTTAGCAGACATTGGAAG-3' and 5'-GTGTGTGCTAACCGTTACCT-3').

\section{Statistical analysis}

All experiments were repeated at least three times. Significant differences between treatment groups were analyzed by analysis of variance (ANOVA) (Statview, Abacus Concept, Berkeley, CA, USA). A $P$ value of $<0.05$ was considered statistically significant.

\section{Results}

S1P induces VEGF-C mRNA and protein expression in HUVECs

Our previous studies found that lysophosphatidic acid (LPA) enhances the expression of VEGF-C in HUVECs ${ }^{[7,8]}$. We further investigated if S1P, a lysophospholipid with similar structure and functions as LPA, also upregulates VEGF-C expression in endothelial cells. Cells were serum-starved for $12 \mathrm{~h}$ in M199 medium, followed by S1P treatment for $4 \mathrm{~h}$ at the indicated concentrations. It was observed that S1P upregulated VEGF-C mRNA expression in HUVECs in a concentrationdependent manner (Figure 1A). Induction of VEGF-C mRNA expression was observed at $1 \mu \mathrm{mol} / \mathrm{L} \mathrm{S1P}$ and it peaked at 5 $\mu \mathrm{mol} / \mathrm{L}$, with sustained effect at $10 \mu \mathrm{mol} / \mathrm{L}$. Next, we investigated whether S1P-upregulated VEGF-C mRNA expression was time-dependent. HUVECs were incubated with $5 \mu \mathrm{mol} / \mathrm{L} \mathrm{S1P}$ for the indicated time intervals. Enhanced VEGF-C mRNA expression in HUVECs was observed as early as $2 \mathrm{~h}$ after S1P treatment and it peaked at $4 \mathrm{~h}$, after which the enhancement effect declined (Figure 1B). Since VEGF-C mRNA levels increased with S1P treatment, we also investigated if the elevated VEGF-C mRNA levels correlated with protein expression. Total VEGF-C protein levels in HUVECs were detected by ELISA. Our results showed that S1P upregulated VEGF-C protein expression in a concentration- and time-dependent manner (Figure 1C, 1D), consistent with realtime PCR results. These results indicated that S1P enhances VEGF-C mRNA and protein expression in HUVECs.

S1P-induced VEGF-C expression in HUVECs is mediated by an MMP-2-dependent pathway

MMP-2 is an important regulator of angiogenesis. In addition, S1P enhances MMP-2 activity in HUVECs ${ }^{[9]}$. Our previous study demonstrated that S1P-induced HUVEC tube formation was blocked on treatment with an MMP inhibitor and MMP-2 siRNA. Therefore, we further investigated whether MMP-2
A

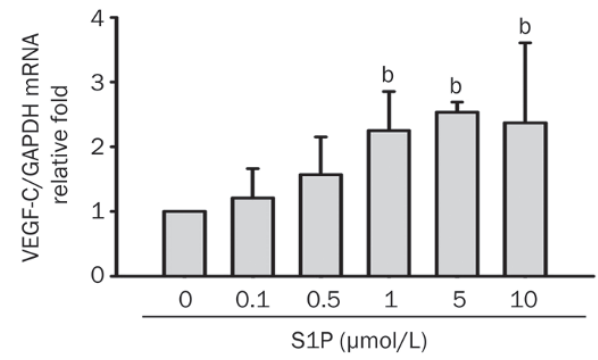

B
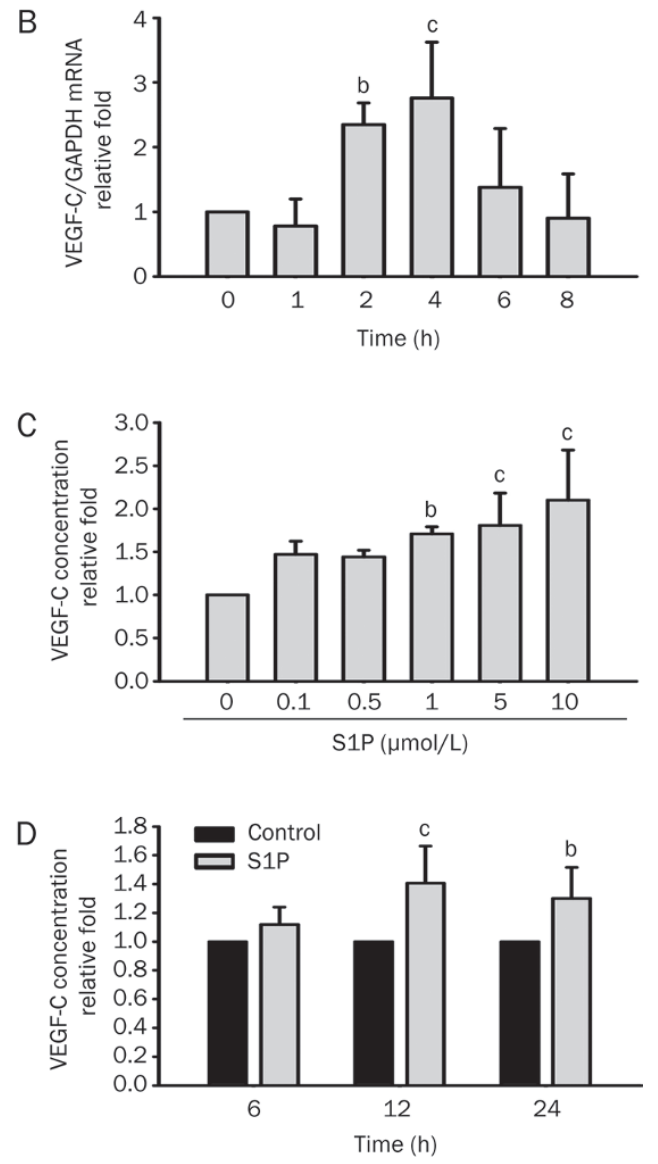

Figure 1. Sphingosine-1-phosphate (S1P) upregulates vascular endothelial growth factor (VEGF)-C expression in human umbilical vein endothelial cells (HUVECs) in a dose- and time-dependent manner. (A) HUVECs were incubated with S1P for $4 \mathrm{~h}$ at the indicated concentrations. RNAs were harvested and analyzed by real-time PCR using specific primer sets for human VEGF-C or GAPDH. (B) HUVECs were incubated with S1P $(5 \mu \mathrm{mol} / \mathrm{L})$ for different time intervals as indicated and subjected to real-time PCR. (C) VEGF-C levels in the supernatant of cultured HUVECs were measured by ELISA after incubation with S1P for $8 \mathrm{~h}$ at various concentrations as indicated. (D) HUVECs were incubated with S1P $(5 \mu \mathrm{mol} / \mathrm{L})$ for the indicated time intervals and VEGF-C levels in the supernatant were measured by ELISA. Quantified results are shown as the mean \pm SD from at least three independent experiments. ${ }^{b} P<0.05$, ${ }^{c} P<0.01$ vs control.

is involved in S1P-induced VEGF-C expression. Pretreatment with GM6001 (10 $\mu \mathrm{mol} / \mathrm{L})$, a broad-spectrum MMP inhibitor, significantly suppressed S1P-induced VEGF-C expression in 
HUVECs (Figure 2A). To further identify the involvement of MMP-2 in VEGF-C induction, cells were transfected with specific or scrambled MMP-2 siRNA by electroporation, followed

A

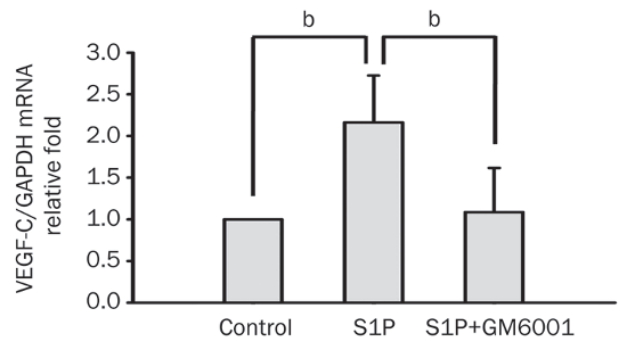

B

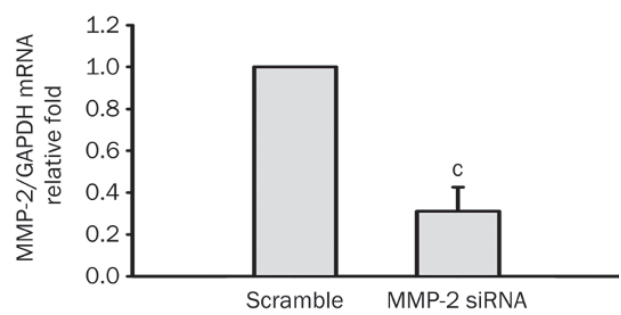

C

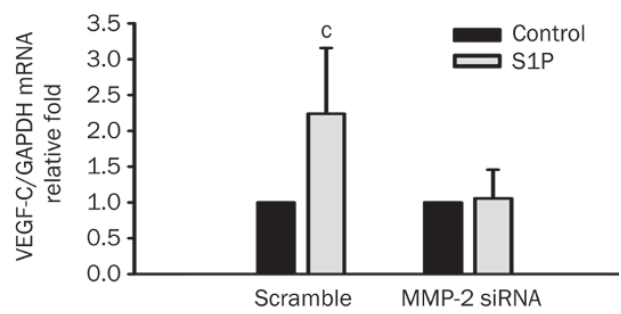

D

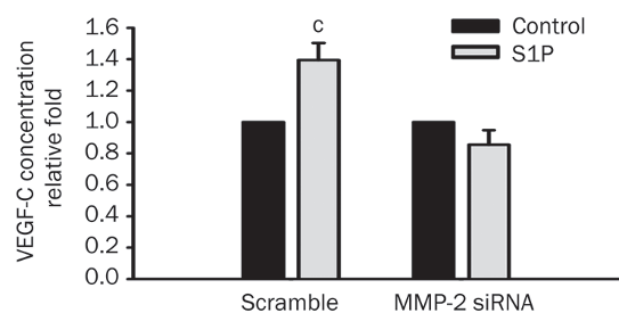

Figure 2. Sphingosine-1-phosphate (S1P) upregulates vascular endothelial growth factor (VEGF)-C expression by a matrix metalloproteinase (MMP)-2-dependent pathway in human umbilical vein endothelial cells (HUVECs). (A) HUVECs were pretreated with GM6001 (10 $\mu \mathrm{mol} / \mathrm{L})$ for $1 \mathrm{~h}$, followed by S1P ( $5 \mu \mathrm{mol} / \mathrm{L})$ treatment for another $4 \mathrm{~h}$. VEGF-C mRNA expression levels were monitored by real-time PCR. (B) siRNA mediated downregulation of MMP-2 mRNA expression in HUVECs. MMP-2 and scrambled siRNA were transfected into HUVECs by electroporation. The transfection efficiency was evaluated by real-time PCR. MMP-2 expression was suppressed in HUVECs transiently transfected with MMP-2 siRNA. (C) HUVECs transfected with scrambled or MMP-2 siRNA were incubated with S1P $(5 \mu \mathrm{mol} / \mathrm{L})$ for $4 \mathrm{~h}$, and VEGF-C mRNA expression levels were monitored by real-time PCR. (D) HUVECs were incubated with S1P (5 $\mu \mathrm{mol} / \mathrm{L}$ ) for $8 \mathrm{~h}$, and the secreted concentration of VEGF-C protein was determined by ELISA. Quantified results are shown as the mean \pm SD from at least three independent experiments. ${ }^{b} P<0.05,{ }^{c} P<0.01$ vs control. by treatment with S1P for $4 \mathrm{~h}$. We found that MMP-2 mRNA expression was knocked down by MMP-2 siRNA in transfected cells (Figure 2B). In addition, S1P-induced VEGF-C mRNA and protein expression was suppressed in MMP-2 knockdown HUVECs (Figure 2C, 2D). In particular, there was inconsistency in VEGF-C induction because of the effects of electroporation and different patient sample batches. These results suggest that S1P-upregulated VEGF-C expression is mediated by an MMP-2-dependent pathway.

S1P-induced VEGF-C expression in HUVECs is mediated by FGFR-1 transactivation

We previously demonstrated that EGFR transactivation was involved in LPA-induced lymphangiogenesis ${ }^{[8]}$. Here we examined whether EGFR also participates in S1P-induced VEGF-C expression. Two receptor inhibitors, AG1478 and SU5402, which respectively are EGFR and FGFR tyrosine kinase inhibitors, were used. We observed that pretreatment with SU5402 $(5 \mu \mathrm{mol} / \mathrm{L})$ for $1 \mathrm{~h}$ significantly suppressed S1Pinduced VEGF-C expression in HUVECs (Figure 3A). In contrast, treatment with AG1478 $(100 \mathrm{nmol} / \mathrm{L})$ had no effect on S1P-induced VEGF-C expression (Figure 3A). This result indicated that S1P-induced VEGF-C expression is FGFR dependent. We next determined the expression profiles of FGFRs (FGFR-1-4) in HUVECs using real-time PCR. FGFR-1 was expressed at the highest levels followed by FGFR- 4 and FGFR-3, whereas FGFR-2 was undetectable (Figure 3B). Since FGFR-1 expression had the highest level among FGFR family members in HUVECs, the extent of involvement of FGFR-1 was further investigated. Cells were first transfected with specific or scrambled FGFR-1 siRNA, followed by treatment with $5 \mu \mathrm{mol} / \mathrm{L} \mathrm{S1P}$ for $4 \mathrm{~h}$. Real-time PCR results revealed that FGFR-1 expression was knocked down by transfection with FGFR-1 siRNA in HUVECs (Figure 3C). Moreover, S1Pinduced VEGF-C mRNA and protein expression was significantly suppressed in FGFR-1-knockdown HUVECs (Figure 3D, 3E). These results demonstrated that S1P-enhanced VEGF-C expression in HUVECs was mediated by FGFR-1.

\section{S1P induces FGFR-1 phosphorylation in HUVECs}

Since S1P-induced VEGF-C expression was FGFR-1 dependent, we further investigated whether S1P activates FGFR-1. Using an FGFR-1-phospho ELISA, we showed that S1P induced FGFR-1 phosphorylation in a time-dependent manner. Phosphorylated FGFR-1 levels peaked at $1 \mathrm{~min}$, with a sustained effect at $5 \mathrm{~min}$ after treatment with $5 \mu \mathrm{mol} / \mathrm{L} \mathrm{S1P}$ (Figure 4A). We next investigated whether MMPs play a role in S1P-enhanced FGFR-1 phosphorylation. Cells were pretreated with GM6001 $(10 \mu \mathrm{mol} / \mathrm{L})$ for $1 \mathrm{~h}$, followed by treatment with S1P $(5 \mu \mathrm{mol} / \mathrm{L})$ for $1 \mathrm{~min}$. The results showed that S1P-induced FGFR-1 phosphorylation was suppressed by pretreatment with GM6001 in HUVECs (Figure 4B). FGF-2stimulated FGFR-1 phosphorylation was used as a positive control. These findings revealed that S1P induces FGFR-1 activation, and MMP might be an essential upstream signal in FGFR-1 transactivation. 

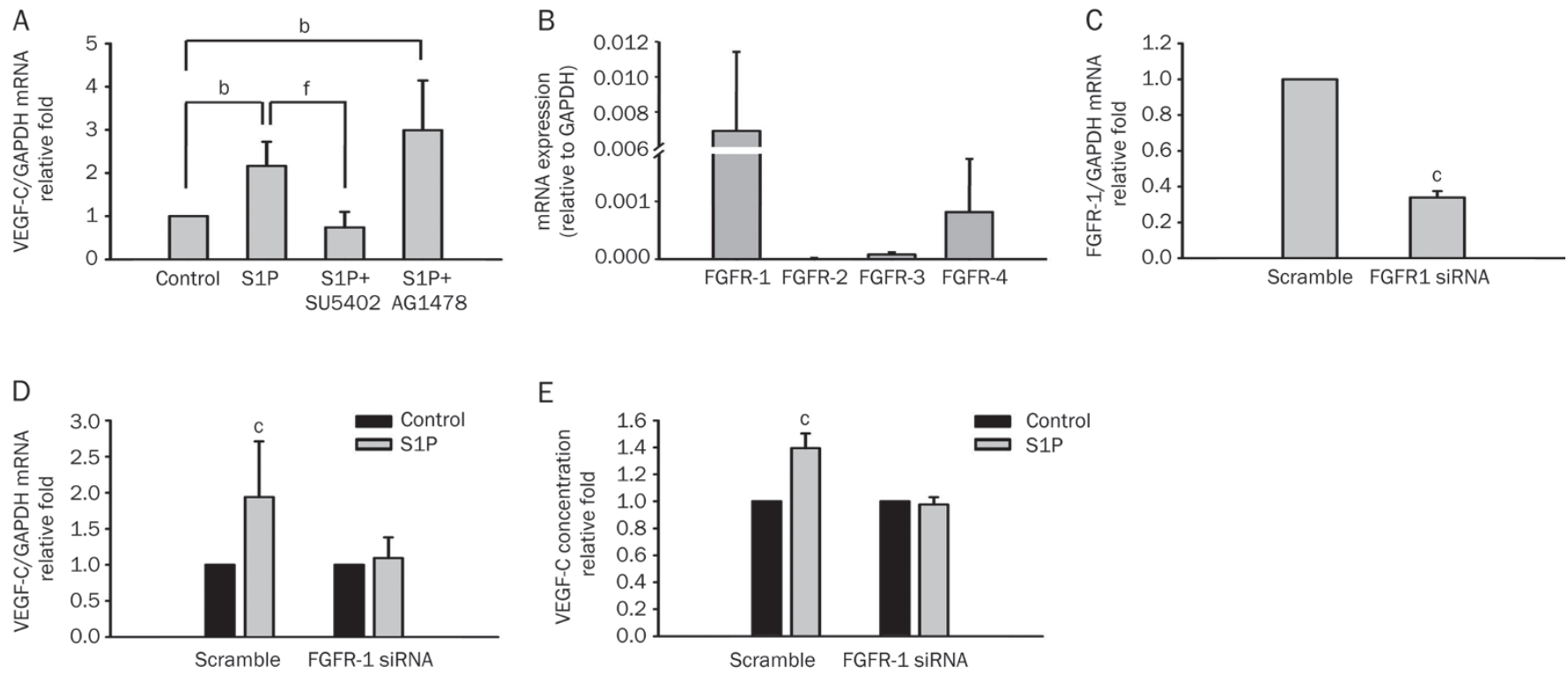

Figure 3. Sphingosine-1-phosphate (S1P) upregulates vascular endothelial growth factor (VEGF)-C expression by a fibroblast growth factor receptor (FGFR)-1-dependent pathway in human umbilical vein endothelial cells (HUVECs). (A) HUVECs were pretreated with SU5402 (5 $\mu$ mol/L) or AG1478 $(100 \mathrm{nmol} / \mathrm{L})$ for $1 \mathrm{~h}$, followed by S1P $(5 \mu \mathrm{mol} / \mathrm{L})$ treatment for another $4 \mathrm{~h}$. VEGF-C mRNA expression levels were monitored by real-time PCR. (B) Total mRNA was extracted from HUVECs, and reverse-transcribed to cDNA. According to real-time PCR, the relative levels of different FGF receptors normalized to GAPDH are shown. (C) FGFR-1 and scrambled siRNA were transfected into HUVECs by electroporation. The transfection efficiency was evaluated by real-time PCR. FGFR-1 expression was suppressed in HUVECs transiently transfected with FGFR-1 siRNA. (D) HUVECs were transfected with scrambled or FGFR-1 siRNA, followed by incubation with S1P $(5 \mu \mathrm{mol} / \mathrm{L})$ for $4 \mathrm{~h}$, and VEGF-C mRNA expression levels were monitored by real-time PCR. (E) HUVECs were incubated with S1P $(5 \mu \mathrm{mol} / \mathrm{L})$ for $8 \mathrm{~h}$, and the secreted concentration of VEGF-C protein was determined by ELISA. Quantified results are shown as the mean $\pm \mathrm{SD}$ from at least three independent experiments. ${ }^{\mathrm{b}} P<0.05,{ }^{\mathrm{C}} P<0.01$ vs control. ${ }^{\mathrm{f}} P<0.01$ vs $\mathrm{S} 1 \mathrm{P}$.

\section{S1P-induced VEGF-C mRNA expression is mediated by FGF-1 but not FGF-2}

As mentioned above, we showed that S1P-induced VEGF-C expression is mediated by FGFR-1. We then determined which FGFs participate in the signaling pathway. HUVECs have been shown to express FGF-1, -2, -5, -7, -8, -11, -12, -16, and $-18^{[10]}$. Since FGF-1 subfamilies are known to have angiogenic properties, we investigated whether FGF-1 and -2 are involved in transactivation. Treatment with FGF-1 shRNA or FGF-2 siRNA was effective in transfected cells (Figure 5A, 5B), and S1P-induced VEGF-C mRNA expression was suppressed by introducing FGF-1 shRNA, but not by FGF-2 siRNA (Figure $5 \mathrm{C}, 5 \mathrm{D})$. These results suggest that S1P-induced VEGF-C expression is mediated by an FGF-1-dependent pathway.

\section{Discussion}

Our previous studies reported that LPA, an LPL with a similar structure and function as S1P, enhanced VEGF-C and lymphatic marker expression through EGFR transactivation in HUVECs ${ }^{[7,8]}$. However, our findings clarified a new S1Pmediated signaling pathway, different from the one mediated by LPA. We found that S1P-induced VEGF-C expression was dependent on the MMP-2, FGF-1, and FGFR-1 transactivationdependent pathway.

It was found that the G-protein-coupled receptor-mediated EGFR transactivation pathway is MMP dependent ${ }^{[8,11]}$. MMPs are zinc-dependent endopeptidases that degrade and regulate many other components of the extracellular matrix (ECM). Importantly, MMPs play key roles in endothelial cell migration and ECM remodeling during angiogenesis ${ }^{[12]}$. They are known to mobilize growth factors by releasing them from the degraded matrix, and hence, modulate multiple cell behaviors $^{[13]}$. For instance, MMPs mobilize and activate VEGF by releasing VEGF-binding ECM proteins ${ }^{[14,15]}$, and also catalyze proteolysis of heparan-sulfate proteoglycan to release FGF-2 in endothelial cells ${ }^{[16]}$. Previous studies also showed that MMP-2 levels and its enzymatic activities were upregulated by LPLs in endothelial cells ${ }^{[9,17]}$. These studies indicated that LPLs may play important roles in endothelial cell invasion by regulating MMP-2 expression. Here we demonstrated that MMP-2 inhibition abrogated S1P-induced VEGF-C expression in HUVECs. These findings suggest that MMP-2 may be activated by S1P in human endothelial cells, thus promoting angiogenesis.

FGFs are a family of heparin-binding growth factors. To date, 23 structurally related members of the FGF family have been identified ${ }^{[18]}$. Among them, the FGF-1 subfamily, including FGF-1 and FGF-2, is the most extensively studied and known for its angiogenesis activity ${ }^{[19]}$. FGF-1 and -2 exert a broad range of proangiogenic activities through binding to high-affinity tyrosine kinase FGFRs on the endothelial cell surface. FGFR-1 is abundantly expressed on endothelial cells in vitro and in vivo ${ }^{[10,20,21]}$, consistent with our results. It triggers cell proliferation and migration as well as protease 

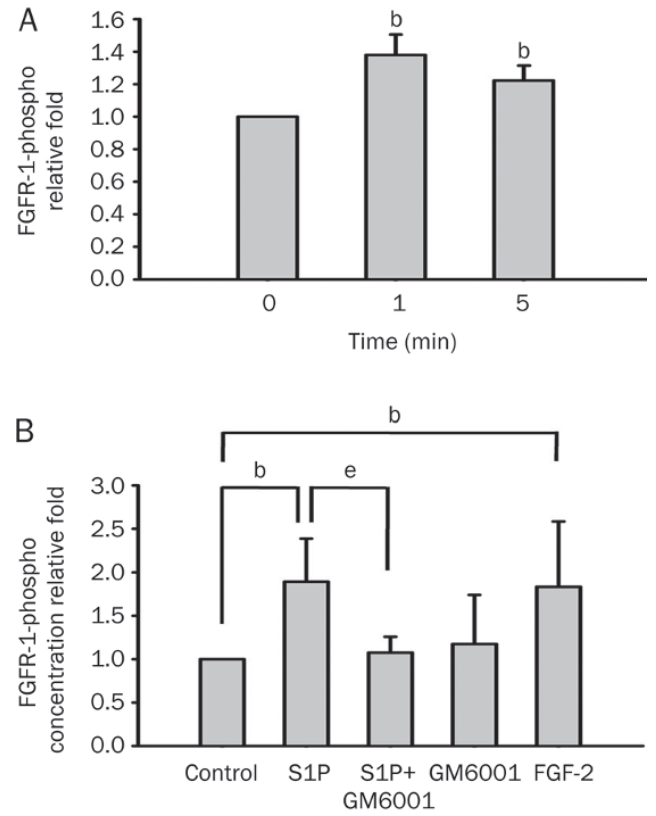

Figure 4. Sphingosine-1-phosphate (S1P) induces fibroblast growth factor (FGF) receptor (FGFR)-1 phosphorylation in a time-dependent manner in human umbilical vein endothelial cells (HUVECs). (A) Starved HUVECs were treated with S1P $(5 \mu \mathrm{mol} / \mathrm{L})$ for 1 and $5 \mathrm{~min}$. The concentration of phosphorylated FGFR-1 was measured by an FGFR-1-phospho ELISA kit. (B) HUVECs were pretreated with GM6001 ( $5 \mu \mathrm{mol} / \mathrm{L}$ ) for $1 \mathrm{~h}$, followed by S1P or FGF-2 treatment for $1 \mathrm{~min}$. The concentration of phosphorylated FGFR-1 was measured by an FGFR-1-phospho ELISA kit. All ELISA data are expressed as the mean $\pm S D$ from at least three independent experiments. ${ }^{\mathrm{b}} \mathrm{P}<0.05$ vs control. ${ }^{\mathrm{e}} \mathrm{P}<0.05$ vs S1P.
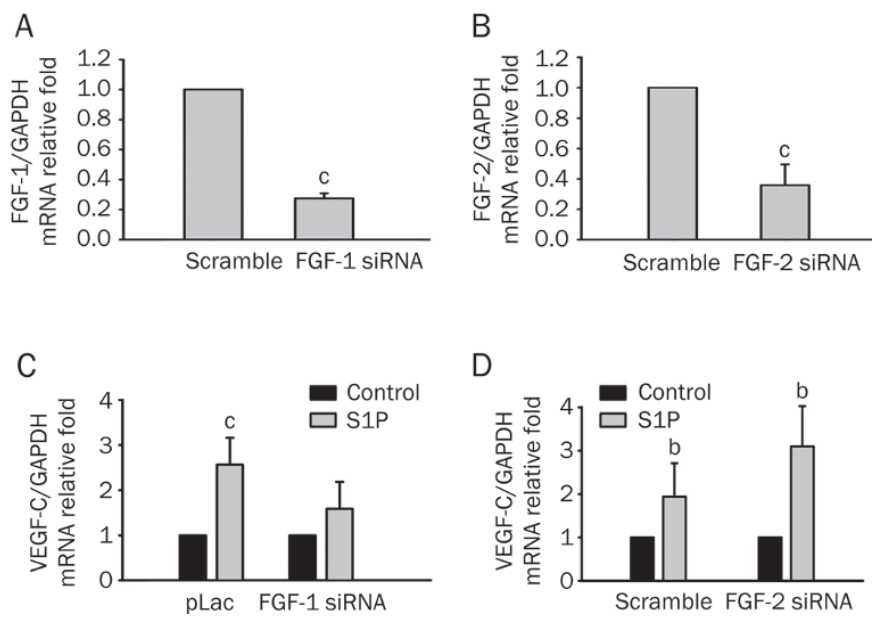

Figure 5. Sphingosine-1-phosphate (S1P)-upregulated vascular endothelial growth factor (VEGF)-C mRNA expression is suppressed by shRNA of fibroblast growth factor (FGF)-1 but not siRNA of FGF-2 in human umbilical vein endothelial cells (HUVECs). HUVECs were transfected with scrambled, FGF-1 shRNA (A), and FGF-2 siRNA (B) by electroporation, and the transfection efficiency was evaluated by real-time PCR. FGF-1 (C) and FGF-2 knockdown (D) HUVECs were incubated with S1P $(5 \mu \mathrm{mol} / \mathrm{L})$ for $4 \mathrm{~h}$, and VEGF-C mRNA expression levels were monitored by real-time PCR. Quantified results are shown as the mean \pm SD from at least three independent experiments. ${ }^{\mathrm{b}} P<0.05,{ }^{\mathrm{c}} P<0.01$ vs control. production upon angiogenic FGF stimulation ${ }^{[20]}$. Blockade of FGFR-1 activity has been shown to inhibit tumor development, angiogenesis, and invasion ${ }^{[22]}$. In addition, FGFR-1 vaccination was also shown to inhibit the growth of tumors in a mouse model ${ }^{[23]}$. These studies suggested that signaling through FGFR-1 is critical for regulating endothelial cellular functions that contribute to vessel maturation, thus facilitating tumor progression. Based on our study, we concluded that FGFR-1 might be essential for S1P's upregulation of VEGF-C expression. Although our result showed FGFR-3 and -4 had relatively low expression levels, their possible effects on S1Pinduced VEGF-C expression require further investigation.

A previous study showed that FGF-2 stimulates both angiogenesis and lymphangiogenesis in a mouse cornea model ${ }^{[24]}$. Furthermore, incubation of endothelial cells with FGF-1 leads to microvascular branching and angiogenesis ${ }^{[25,26]}$. They have also been demonstrated to play a role in regulating blood vessel growth in many pathological conditions by interacting with $\mathrm{VEGFs}^{[27,28]}{ }^{28}$. FGFs bind to heparan sulfate glycosaminoglycan chains attached by the heparan sulfate proteoglycans (HSPGs), which are expressed on ECM of endothelial cells ${ }^{[29]}$. In particular, HSPGs on the cell surface act as a reservoir for FGF-2 ${ }^{[30]}$, which is released in an active form when its binding to ECM is degraded by cellular heparanase ${ }^{[31]}$. It has been reported that FGF-2 can be released from the ECM by MMP-2, thus facilitating cell viability ${ }^{[32]}$. Although most studies focused on FGF-2 and its regulation of binding to ECM, FGF-1 may have similar behaviors to FGF-2. In the present study, we demonstrated that S1P-induced VEGF-C expression depends on FGF-1, and this effect may be caused by MMP-2 activation. However, details of the signaling pathway still need to be further investigated.

In conclusion, this study demonstrated transactivation of S1P enhanced VEGF-C expression in endothelial cells. Our study also clarifies the role of FGFR-1, which participates in S1P-induced VEGF-C expression, and that transactivation is mediated by MMP-2 and FGF-1.

\section{Acknowledgements}

The research was supported by two grants, NSC100-2325-B002-045- from the National Science Council and NHRI-EX10110130BI from the National Health Research Institutes of Taiwan, China.

\section{Author contribution}

Hsinyu LEE and JenHer LU designed research; Chi-hao CHANG, Yuan-li HUANG, and Chi-hsin LIN performed research; JenHer LU, Tsai-kai JU, Ming-kwang SHYU, and Shee-uan CHEN contributed reagents, materials, and analytic tools; JenHer Lu and Hsinyu LEE analyzed data; and Chi-hao CHANG and Hsinyu LEE wrote the paper.

\section{References}

1 Yatomi Y, Igarashi Y, Yang L, Hisano N, Qi R, Asazuma N, et al. Sphingosine 1-phosphate, a bioactive sphingolipid abundantly stored in platelets, is a normal constituent of human plasma and serum. J 
Biochem 1997; 121: 969-73.

2 Spiegel S, Milstien S. Sphingosine-1-phosphate: an enigmatic signaling lipid. Nat Rev Mol Cell Biol 2003; 4: 397-407.

3 Sakurai T, Kudo M. Signaling pathways governing tumor angiogenesis. Oncology 2011; 81: 24-9.

4 Skobe M, Hamberg LM, Hawighorst T, Schirner M, Wolf GL, Alitalo K, et al. Concurrent induction of lymphangiogenesis, angiogenesis, and macrophage recruitment by vascular endothelial growth factor- $\mathrm{C}$ in melanoma. Am J Pathol 2001; 159: 893-903.

5 Lee OH, Kim YM, Lee YM, Moon EJ, Lee DJ, Kim JH, et al. Sphingosine 1-phosphate induces angiogenesis: its angiogenic action and signaling mechanism in human umbilical vein endothelial cells. Biochem Biophys Res Commun 1999; 264: 743-50.

6 Visentin B, Vekich JA, Sibbald BJ, Cavalli AL, Moreno KM, Matteo RG, et al. Validation of an anti-sphingosine-1-phosphate antibody as a potential therapeutic in reducing growth, invasion, and angiogenesis in multiple tumor lineages. Cancer Cell 2006; 9: 225-38.

7 Lin Cl, Chen CN, Huang MT, Lee SJ, Lin CH, Chang CC, et al. Lysophosphatidic acid up-regulates vascular endothelial growth factor- $\mathrm{C}$ and lymphatic marker expressions in human endothelial cells. Cell Mol Life Sci 2008; 65: 2740-51.

8 Lin Cl, Chen CN, Huang MT, Lee SJ, Lin CH, Chang CC, et al. Lysophosphatidic acid upregulates vascular endothelial growth factor- $\mathrm{C}$ and tube formation in human endothelial cells through $\operatorname{LPA}(1 / 3), \operatorname{COX}-2$, and NF-kappaB activation- and EGFR transactivation-dependent mechanisms. Cell Signal 2008; 20: 1804-14.

9 Wu WT, Chen CN, Lin Cl, Chen JH, Lee H. Lysophospholipids enhance matrix metalloproteinase- 2 expression in human endothelial cells. Endocrinology 2005; 146: 3387-400.

10 Antoine M, Wirz W, Tag CG, Mavituna M, Emans N, Korff T, et al. Expression pattern of fibroblast growth factors (FGFs), their receptors and antagonists in primary endothelial cells and vascular smooth muscle cells. Growth Factors 2005; 23: 87-95.

11 Shah BH, Catt KJ. GPCR-mediated transactivation of RTKs in the CNS: mechanisms and consequences. Trends Neurosci 2004; 27: 48-53.

12 Nagase H, Visse R, Murphy G. Structure and function of matrix metalloproteinases and TIMPs. Cardiovasc Res 2006; 69: 562-73.

13 Rodriguez D, Morrison CJ, Overall CM. Matrix metalloproteinases: what do they not do? New substrates and biological roles identified by murine models and proteomics. Biochim Biophys Acta 2010; 1803: 39-54.

14 Dean RA, Butler GS, Hamma-Kourbali Y, Delbé J, Brigstock DR, Courty $\mathrm{J}$, et al. Identification of candidate angiogenic inhibitors processed by matrix metalloproteinase 2 (MMP-2) in cell-based proteomic screens: disruption of vascular endothelial growth factor (VEGF)/heparin affin regulatory peptide (pleiotrophin) and VEGF/Connective tissue growth factor angiogenic inhibitory complexes by MMP-2 proteolysis. Mol Cell Biol 2007; 27: 8454-65.

15 Lee S, Jilani SM, Nikolova GV, Carpizo D, Iruela-Arispe ML. Processing of VEGF-A by matrix metalloproteinases regulates bioavailability and vascular patterning in tumors. J Cell Biol 2005; 169: 681-91.

16 Whitelock JM, Murdoch AD, lozzo RV, Underwood PA. The degradation of human endothelial cell-derived perlecan and release of bound basic fibroblast growth factor by stromelysin, collagenase, plasmin, and heparanases. J Biol Chem 1996; 271: 10079-86.

17 Sun HY, Wei SP, Xu RC, Xu PX, Zhang WC. Sphingosine-1-phosphate induces human endothelial VEGF and MMP-2 production via transcription factor ZNF580: novel insights into angiogenesis. Biochem Biophys Res Commun 2010; 395: 361-6.

18 Powers CJ, McLeskey SW, Wellstein A. Fibroblast growth factors, their receptors and signaling. Endocr Relat Cancer 2000; 7: 165-97.

19 Beenken A, Mohammadi M. The FGF family: biology, pathophysiology and therapy. Nat Rev Drug Discov 2009; 8: 235-53.

20 Javerzat S, Auguste P, Bikfalvi A. The role of fibroblast growth factors in vascular development. Trends Mol Med 2002; 8: 483-9.

21 Hughes SE. Differential expression of the fibroblast growth factor receptor (FGFR) multigene family in normal human adult tissues. J Histochem Cytochem 1997; 45: 1005-19.

22 Rousseau B, Larrieu-Lahargue F, Javerzat S, Guilhem-Ducléon F, Beermann F, Bikfalvi A. The tyrp1-Tag/tyrp1-FGFR1-DN bigenic mouse: a model for selective inhibition of tumor development, angiogenesis, and invasion into the neural tissue by blockade of fibroblast growth factor receptor activity. Cancer Res 2004; 64: 2490-5.

23 He QM, Wei YQ, Tian L, Zhao X, Su JM, Yang L, et al. Inhibition of tumor growth with a vaccine based on xenogeneic homologous fibroblast growth factor receptor-1 in mice. J Biol Chem 2003; 278 : 21831-6.

24 Cao R, Eriksson A, Kubo H, Alitalo K, Cao Y, Thyberg J. Comparative evaluation of FGF-2-, VEGF-A-, and VEGF-C-induced angiogenesis, lymphangiogenesis, vascular fenestrations, and permeability. Circ Res 2004; 94: 664-70.

25 Uriel S, Brey EM, Greisler HP. Sustained low levels of fibroblast growth factor-1 promote persistent microvascular network formation. Am J Surg 2006; 192: 604-9.

26 Xue L, Greisler HP. Angiogenic effect of fibroblast growth factor-1 and vascular endothelial growth factor and their synergism in a novel in vitro quantitative fibrin-based 3-dimensional angiogenesis system. Surgery 2002; 132: 259-67.

27 Chang LK, Garcia-Cardeña G, Farnebo F, Fannon M, Chen EJ, Butterfield C, et al. Dose-dependent response of FGF-2 for lymphangiogenesis. Proc Natl Acad Sci U S A 2004; 101: 11658-63.

28 Tomanek RJ, Sandra A, Zheng W, Brock T, Bjercke RJ, Holifield JS. Vascular endothelial growth factor and basic fibroblast growth factor differentially modulate early postnatal coronary angiogenesis. Circ Res 2001; 88: 1135-41.

29 Presta M, Dell'Era P, Mitola S, Moroni E, Ronca R, Rusnati M. Fibroblast growth factor/fibroblast growth factor receptor system in angiogenesis. Cytokine Growth Factor Rev 2005; 16: 159-78.

30 Presta M, Maier JA, Rusnati M, Ragnotti G. Basic fibroblast growth factor is released from endothelial extracellular matrix in a biologically active form. J Cell Physiol 1989; 140: 68-74.

31 Vlodavsky I, Korner G, Ishai-Michaeli R, Bashkin P, Bar-Shavit R, Fuks Z. Extracellular matrix-resident growth factors and enzymes: possible involvement in tumor metastasis and angiogenesis. Cancer Metastasis Rev 1990; 9: 203-26.

32 Tholozan FM, Gribbon C, Li Z, Goldberg MW, Prescott AR, McKie N, et al. FGF-2 release from the lens capsule by MMP-2 maintains lens epithelial cell viability. Mol Biol Cell 2007; 18: 4222-31. 